ANUARIO DE ESTUDIOS MEDIEVALES

42/2, julio-diciembre de 2012, pp. 469-478

ISSN 0066-5061

doi:10.3989/aem.2012.42.2.07

\title{
DE NUEVO SOBRE EL CARMEN IN LAUDEM RAIMUNDI BERENGARII IV DEL MANUSCRITO 31 DE RODA (OLIM 8) ${ }^{1}$
}

\author{
THE CARMEN IN LAUDEM RAIMUNDI BERENGARII IV \\ IN MANUSCRIPT 31 OF RODA (OLIM 8): A NEW APPROACH
}

\author{
Jesús Alturo I PERUCHO \\ Universitat Autònoma de Barcelona
}

\begin{abstract}
Resumen: Nueva edición crítica del Carmen in laudem Raimundi Berengarii IV, con nuevas hipótesis sobre la fecha y lugar de su composición (Lérida, inmediatamente después de su reconquista en 1149) y posible atribución de su autoría al canónigo de la catedral de Barcelona y notario de la cancillería condal Poncio.
\end{abstract}

Palabras clave: Ramón Berenguer IV, conde de Barcelona; poesía latina medieval civil.

\begin{abstract}
This is a new critical edition of Carmen in laudem Raimundi Berengarii $I V$, with new hypotheses about the date and place of its composition (Lérida, immediately after its reconquest in 1149) and possible attribution of authorship to Poncio, canon of the Cathedral of Barcelona and notary of the Count Chancellery.
\end{abstract}

Keywords: Ramón Berenguer IV, count of Barcelona; medieval Latin civil poetry.

El conde de Barcelona Ramón Berenguer IV fue celebrado por los poetas a su muerte ${ }^{2}$, pero, ya en vida, tuvo a su servicio vates que ensalzaron sus glorias. $Y$ es que el príncipe de Aragón no era insensible a los elogios ${ }^{3}$, ni pretería las ventajas

\footnotetext{
${ }^{1}$ Este estudio se ha realizado en el marco del proyecto de investigación HAR2009-11367 concedido por el Ministerio de Ciencia e Innovación, que dirijo.

${ }^{2}$ Recuérdense los títulos de gloria que se le atribuyen en su epitafio en pergamino, tal como lo publicaron A. Merino; J. de la Canal, España Sagrada, p. 466: "Hic certe rex pacis, princeps iustitiae, dux ueritatis et aequitatis, armiger intemeratae fidei christianae, contra Sarracenos et infideles debelator fortis". Por otra parte, según P. de Bofarull, Los condes de Barcelona vindicados, p. 200, en su sepulcro se podía leer la siguiente inscripción: "Dux ego de matre, Rex coniuge, Marchio patre: / Marte, fame fregi mauros, dum tempore degi./ Et sine iactura, tenui Domino sua iura". Además, se le dedicó un "epicedion", transmitido en el último folio del ms. lat. 5132 de la Biblioteca Nacional de Francia, editado por Ll. Nicolau d'Olwer, L'escola poètica de Ripoll, pp. 36-38, e incorporado también en el disco de J. Savall, Mentem meam ledit dolor.

3 J.E. Ruiz-Domènec, España, pp. 221-222, incluso ve en él un vanidoso, aunque reconoce su habilidad política. En general, el juicio que Ramón Berenguer ha merecido a los historiadores es positivo, puesto que le avalan sus éxitos bélicos y políticos. Véase al respecto el repaso de opiniones que hace F. Rodríguez Bernal, Ramón Berenguer IV, pp. 25-46. Añadamos, por lo demás, que parece cierta la afirmación de F. Soldevila, Ramón Berenguer IV, pp. 29-30, de que el ideal de Ramón Berenguer IV "era, indubtablement, la formació del gran reialme pirinenc occitano-peninsular", por lo que podemos ver en él "el gran plasmador de Catalunya", puesto que "les seves grans conquestes foren adreçades a completar el Principat de Catalunya". De
} 
de la propaganda escrita u oral ya fuera en su favor o en el de sus antepasados. No en vano la primera versión de los Gesta comitum Barcinonensium se inició, según mi opinión, probablemente, ya bajo su gobierno ${ }^{4}$, como nueva prueba, si así fuera, de su evidente habilidad política al lado de sus incuestionables dotes militares, cualidades que se tradujeron en éxitos que hicieron trascender su fama allende las fronteras de sus dominios directos. Su biógrafo Ferran Soldevila, que se ocupó de su reputación entre los grandes personajes de su tiempo ${ }^{5}$, de su glorificación en la leyen-

ahí que Soldevila llegue a verlo no sólo "com una de les més grans figures entre els comtes de Barcelona des de Guifré I, sinó també com una de les més grans entre els comtes-reis de Catalunya-Aragó", p. 21. Por su parte, P.E. Schramm, en su breve, pero enjundioso estudio Ramon Berenguer IV, p. 41, resalta que: "Des del punt de vista del poder polític, el regne de Ramon Berenguer provocà un desplaçament d'equilibri en tota la Península (...). En el moment en què Ramon Berenguer havia succeït el seu pare, les potències principals de la Península eren el reialme navarro-aragonès del Batallador $\mathrm{i}$ «l'Imperium» d'Alfons VII, que s'estenia des de Saragossa fins a Galícia i de la Rioja fins a Toledo. Quan Ramon Berenguer es va morir, ell era la figura més poderosa del joc de forces d'Espanya, malgrat que hagués de reconèixer un sobirà secular i un altre d'eclesiàstic".

${ }^{4}$ A mi modo de ver, la primera de las diversas etapas de la elaboración de esta obra ya se comenzó en vida de Ramón Berenguer IV y a instancias suyas, y habría sido compuesta en su mismo entorno cancilleresco, no en el monasterio de Ripoll. Incluso intuyo que su primer autor pudo ser el canónigo de la catedral de Barcelona y, a la vez, notario de la cancillería condal Poncio. Adelanto ya esta idea, con todas las cautelas posibles, en J. Alturo, La historiografia catalana del període primitiu, pp. 27-28. Espero poder desarrollar pronto esta cuestión más detenidamente, aunque no se me oculta que la opinión, hoy por hoy, todavía más generalizada es la que considera los Gesta como un texto compuesto inicialmente en el citado monasterio pirenaico y en tiempos de Alfonso el Casto. Así lo mantiene, por ejemplo, quien en la actualidad mayor dedicación destina a esta cuestión, S.M. Cingolani, del que, entre su extensa bibliografía sobre el tema, baste citar ahora S.M. Cingolani, Gestes dels Comtes de Barcelona. No es éste el lugar para abordar esta problemática; pero recordemos, simplemente, que el hecho de que un códice se haya conservado en la biblioteca de un centro monástico, no implica, por necesidad, que los textos que contenga, aunque sean únicos, se compusieran en ese mismo lugar. Piénsese, sin ir más lejos, en los célebres Carmina Riuipullensia, copiados y conservados en Ripoll, pero no compuestos allí. Por otra parte, en caso de haberse realizado la primera redacción de los Gesta en Ripoll, se diría que, si no todas, buena parte de las notas marginales que recuerdan la vinculación de los condes de Barcelona con el monasterio se hubieran incorporado al texto principal desde el primer momento. Todo ello sin tener en cuenta, desde el punto de vista codicográfico, el carácter meramente "marginal" de la inclusión de esta primera redacción en unos folios del mencionado manuscrito parisino lat. 5132 dejados inicialmente en blanco (ff. 23v-25v), fruto, por otra parte, de diversos amanuenses de épocas distintas, que reflejan, según creo, estadios de redacción textual diferentes. Y el primer cambio de mano se da al comienzo del f. $24 \mathrm{v}$, inmediatamente después de mencionar la obtención del reino de Aragón por Ramón Berenguer debido a su compromiso de boda con la hija del rey Ramiro, pero antes de completar el resto de noticias sobre sus "gestas" hasta llegar al momento de su inhumación, lo que me parece significativo respecto a la fecha de redacción de la primera versión en el sentido que ya he sugerido.

${ }^{5}$ F. Soldevila, Ramon Berenguer IV, pp. 45-46. Véase, a título de ejemplo, los elogios que le dedica el emperador Federico I Barbarroja en el diploma en que confirma el condado de Provenza a su hermano Ramón: "Verum per presentes apices ad noticiam uniuersorum Imperii fidelium deducere et dignum duximus palam omnibus declarare quam sincera, quam diligens, quam prona et quam feruens circa gloriam et honorem Imperii et circa nostram dileccionem fides et deuocio nostri charissimi Raimundi, Barchinonensis comitis et illustrissimi principis, extiterit. Et re uera magnifica eius obsequia et preclara opera subsecuta apercius declarassent quante fidei ac deuocionis circa nostram personam fuerit, nisi, diuina uocante gracia, que aufert spiritum principum, de medio ipse sublatus fuisset; de cuius indigno obitu non possumus in perpetuum non dolere. Huc accedit quod nostra precordialis dileccio erga talem ac tantum amicum ociosa esse nullatenus potuit, presertim cum eius sincerum amorem et studium circa personam et honorem charissime neptis nostre Richildis, Hispaniarum regine, oculata fide perspeximus et ipso rerum affectu ueraciter ita esse probauimus". Este texto ha sido publicado en diversas ocasiones, como en H. Appelt, Friderici I. Diplomata, doc. 382. Al ser incluido en el Liber Feudorum Maior (f. 379), también lo editó F.M. Rosell, Liber Feudorum Maior, 
$\mathrm{da}^{6}$, de su estimación entre los historiadores ${ }^{7}$ y de su enaltecimiento en la literatura ${ }^{8}$, destacó específicamente el alt prestigi que va arribar a aconseguir en els seus regnes $i$ en els regnes estranys ${ }^{9}$. Y así parece, en efecto, que lo pruebe, entre otros testimonios, el carmen compuesto en su honor, que se conserva copiado parcialmente en un folio en blanco, el 232v, del actual códice 31 de Roda (olim 8), custodiado en el Archivo de la Catedral de Lérida.

Las circunstancias que motivaron este poema son fácilmente deducibles a partir de la consideración de su mismo contenido, que nos sitúa a continuación de la reconquista de Tortosa y de Lérida. Su composición remonta, probablemente, a mediados del siglo XII, tras la inmediata recuperación de la capital del Segre. Como es lógico, grosso modo ya lo han visto así quienes se han ocupado de esta cuestión con anterioridad, particularmente Dolores Porta, quien le ha dedicado el más detallado análisis de contextualización histórica ${ }^{10}$. Por lo que respecta a la fecha de su composición, esta investigadora, habida cuenta de que considera que en el poema no se hace ninguna alusión al matrimonio del conde con Petronila, piensa en una data anterior al mes de septiembre de $1150^{11}$, cuando, según ella, dicho matrimonio se celebró, precisamente en Lérida ${ }^{12}$.

doc. 902, y, antes, a partir de esta misma fuente, lo había transcrito G. Pujades, Crónica Universal del Principado, pp. 516-518. A estas alabanzas se pueden añadir las que le dedica el anónimo autor de la primera redacción de los Gesta: "Hic Raimundus Berengarii quartus mira probitate, scientia, ingenio ac consilio pollens, toto orbe famosissimus claruit". Véase L. Barrau; J. Massó, Gesta comitum Barcinonensium, p. 8.

${ }^{6}$ F. Soldevila, Ramon Berenguer IV, pp. 46-52.

${ }^{7}$ Ibidem, pp. 52-55; F. Rodríguez Bernal, Ramón Berenguer IV, y nota 3.

${ }^{8}$ F. Soldevila, Ramon Berenguer IV, pp. 55-71, aunque, en este apartado, no menciona la novela Vassall d'amor de Alfons Roure, por lo que afirma: "Tampoc no ho ha estat [afortunado] pel que fa al ressò de les seves gestes dins la literatura moderna de Catalunya. No manquen composicions floralesques bastides sobre algun episodi de la seva vida, però no sabem que cap gran figura de la nostra poesia -o de la nostra prosa-s'hagi complagut a dur-les al poema heroic, a la novel-la o al teatre. La grandesa de Ramon Berenguer IV no perdura sinó en l'obra dels historiadors", p. 71. Claro que el prolífico autor de sainetes A. Roure i Brugulat (1889-1962) no fue un escritor de primera fila, pero tampoco careció de méritos literarios, ni, menos aún, de imaginación histórico-romántica, fundamentada, por otra parte, en notables conocimientos históricos. He aquí cómo nos describe al conde a sus treinta años: "Era alt $\mathrm{i}$ robust, franc de rostre i d'esguart; ample de pit i de cor; intel-ligent, magnànim i enèrgic. Era hermós, virilment hermós; tenia tota la bellesa física i espiritual d'un home sense nafra, al ple de la seva joventut". Y prosigue, después de recordar que a los diecisiete años accedió al trono, diciendo que "la seva prudència i rectitut en el govern, l'hi dongueren renom de justicier i sapient en tota la cristiandat", pp. 36-37.

${ }^{9}$ F. Soldevila, Ramon Berenguer IV, p. 39. D. Porta, El poema de Roda, pp. 297-310, trae a colación diversos documentos expedidos en territorio zaragozano y oscense que se hacen eco de la conquista de Tortosa y Lérida, y afirma: "Raro es el fondo documental aragonés en el que no figura algún documento con menciones de las conquistas de Tortosa y Lérida. El acontecimiento tuvo resonancia hasta en la extrema zona occidental aragonesa e incluso en comarcas sometidas por el monarca navarro", p. 306.

${ }^{10}$ D. Porta, por su parte, ve en el poema "una acusada intención religiosa", por lo que "más que un poema épico parece un himno religioso", D. Porta, El poema de Roda, p. 300. Es evidente, sin embargo, que la atmósfera religiosa del poema está, ante todo, al servicio de un mayor enaltecimiento político de la figura del conde, pero se inscribe en el ambiente que se respiraba en tiempos de la Segunda Cruzada (1147-1149), años en que, junto con Lérida y Fraga (1149), también se recuperó para la Cristiandad Almería y Lisboa (1147) y la mencionada Tortosa (1148).

${ }_{11}$ Más cauteloso, M. Coll i Alentorn, La historiografia de Catalunya, p. 46, considera como fechas extremas 1149 y 1162, año de la muerte del conde catalán, "puix que sembla ésser-hi al·ludit com a vivent encara".

12 Téngase en cuenta, sin embargo, que, según la documentación aragonesa, el matrimonio tuvo lugar en 1151, aunque, en cualquier caso, antes de febrero de ese año. Véanse los ejemplos aducidos 
Con todo, no debemos pasar por alto el verso penetrasti regale solium, que, aunque va inmediatamente después del hemistiquio rex Illerdensium, no creo que haga referencia a su toma de posesión del reino de Lérida, sino del de Aragón, en cuyo solio real se sentó justamente por su matrimonio con Petronila, a pesar de que, como es bien sabido, nunca usó el título de rey de Aragón. Por ello será preciso concluir que el citado verso alude aquí, en efecto, a su reinado aragonés, pero, en este momento, simplemente por el compromiso de boda in futurum acordado con Ramiro el Monje el 11 de agosto de 1137 en Barbastro ${ }^{13}$.

Por ello creo que podemos dar la razón a Porta y deducir la fecha de composición del poema entre finales de octubre de 1149 y el momento de la boda, posiblemente a principios de $1151^{14}$, como datas extremas. Pero quizá se pueda precisar más. El hecho de que no aparezca tampoco ninguna alusión en el poema a la recuperación cristiana de Fraga y de Mequinenza, tal vez podría interpretarse como prueba de que el poema se compuso en Lérida mismo, acto seguido de su conquista ${ }^{15}$. Por otra parte, como bien observó D. Porta, la intitulación "rey de los leridanos" no fue usada tampoco por Ramón Berenguer. Este título, que se le atribuye en el poema, se explica sólo en un momento muy cercano a la reconquista de la ciudad.

Es una posibilidad más, que, en todo caso, tendría consecuencias en orden a decidir sobre otra cuestión de interés, la del origen del anónimo autor del poema, para el que se ha supuesto un clérigo de Roda; posibilidad, en principio, razonable por cuanto el códice único en que se ha conservado la copia es rotense. Aunque, como enseguida veremos, no creo que tal supuesto origen del autor sea del todo incuestionable, pese a ser ésta la opinión más extendida entre los historiadores.

De hecho, si su composición tuvo lugar, efectivamente, en Roda, podríamos pensar, por supuesto, que su autoría se debiera a algún canónigo rotense. El motivo bien podría ser su agradecimiento por ver alejado el peligro sarraceno; contrariamente a lo que se ha afirmado ${ }^{16}$, veo más difícil pensar que se debiera al hecho de ver amplia-

por R. del Arco, Referencias a acontecimientos históricos, pp. 346-347, y, en particular, p. 346, donde se dice: "Facta carta in mense Februarii era MCLXXXVIIII, eodem anno quando comes Barchinonensis presit uxorem ad illa regina de Aragon".

${ }^{13}$ R. de Huesca, Teatro histórico de las Iglesias, afirma que el rey: "Desde que puso las riendas del gobierno en manos del Conde de Barcelona no se mezcló en asuntos de importancia. Los tratados de alianza, de paz, y de guerra todos se hicieron, y se autorizaron por solo el Conde, sin que suene el nombre de Don Ramiro". Y aún prosigue: "La perfecta armonia que hubo entre suegro y yerno, sin ofrecer motivo de discordia en tantos años, sería de admirar entre padre e hijo, puestos en iguales circunstancias", p. 188. Por otra parte, el P.R. de Huesca, que considera "Don Ramon Berenguer, Conde de Barcelona, uno de los Príncipes más valerosos y prudentes de su siglo”, p. 187, también sitúa su boda con Petronila en el año 1151, p. 188. Por lo demás, de los muchos cronogramas de diplomas aragoneses de la época que hacen referencia al "reinado" efectivo de Ramón Berenguer en Aragón baste citar un par de ejemplos: en una venta de junio de 1139 se lee: "Regnante Ranimirus rex et comes Barquilonensis in Aragon et Superarbi et Ripacurzia", doc. 180 de A. Martín Duque, Colección diplomática; y en una carta de ingenuidad de enero de 1155: "Regnante comite Barchinonensi et princeps Aragonensium", doc. 192.

14 Véase la nota 12.

15 Obsérvese que el Chronicon Rotense, éste sí producido sin duda en Roda, no olvida -como tampoco el resto de anales catalanes- mencionar la conquista por parte de Ramón Berenguer de Fraga, al lado de la de Lérida, Tortosa y Siurana. Dice este texto: "Anno MCLXII obiit Raimundus Berengarius, Comes Barchinonensium et Princeps Aragonensium, qui cepit Ilerdam et Fragam et Tortosam et Siuranam cum omnibus finibus earum". Véase J. Villanueva, Viage literario, p. 334, y, de manera más general, F. Bautista, Breve historiografía, pp. 113-190.

${ }^{16}$ D. Porta, El poema de Roda, p. 307. 
dos los límites territoriales de su diócesis ${ }^{17}$, puesto que la sede episcopal pasó entonces a Lérida ${ }^{18}$, y en Roda quedó sólo una comunidad canonical, si bien con derecho a voto en la elección del obispo de la nueva sede ilerdense ${ }^{19}$.

Y, naturalmente, sea cual sea el origen del poeta, debemos suponerle, asimismo, la suficiente capacidad intelectual y preparación literaria para componer un poema, que, aunque sencillo, no sólo es correcto, sino que no carece de mérito artístico en su misma simplicidad, por lo que se lee con gusto. Por lo demás, en este punto, debemos resaltar que los errores textuales que contiene la copia de este poema descartan la posibilidad de que ésta sea la original y que pueda atribuirse a su mismo autor, aunque se deba, muy probablemente, a un contemporáneo suyo, puesto que su escritura parece propia de finales de la duodécima centuria.

De tratarse, por lo demás, de un autor del entorno rotense quizá tendríamos que pensar en primer lugar en el canónigo Elías, escritor de probados méritos literarios, como se ve en su Vita beati Raimundi episcopi Rotensis, compuesta en 1138, y, muy posiblemente, autor también del Officium del mismo santo prelado. Pero, aunque los historiadores acostumbren referirse a Elias como canónigo de Roda ${ }^{20}$, muy probablemente, éste lo era de Barbastro ${ }^{21}$, sede a la que, como acabo de recordar, se unió la de Roda después de que aquella ciudad fuera reconquistada por Pedro de Aragón, sin que Roda perdiera por ello su título de catedral.

Descartado, pues, Elías, por otra parte, poco testimoniado en la documentación conocida, y continuando con la hipótesis de un autor rotense, no podemos dejar de pensar tampoco en Arnaldo de Coll como uno de sus posibles autores; y es que este canónigo, de acuerdo con la documentación hoy disponible, redactó los más importantes textos diplomáticos de Roda durante la supuesta época de composición de la pieza que nos ocupa; siempre con notable capacidad lingüística y aún cierta pericia literaria ${ }^{22}$. Pero no parecen faltar argumentos, como los que aduzco más abajo, para

17 Recordemos al respecto las palabras del P.R. de Huesca, Teatro histórico de las Iglesias, p. 206: "El pueblo de Roda perdió con la sede el título de ciudad (...) pero fue incomparablemente mayor la pérdida de su iglesia, porque el Obispo Guillermo se llevó con la sede lo más rico y precioso que había en ella, las rentas y derechos Episcopales, y tres Arcedianatos también con sus rentas (...). En la Iglesia de Roda quedaron las Dignidades de Prior mayor, Camarero, Enfermero, Limosnero y Sacristán, y los Canónigos, cuyo número era indefinido por ser Iglesia regular y recepticia”.

${ }_{18} \mathrm{Y}$ no olvidemos que estaba en Barbastro desde su reconquista en 1101, con los paréntesis de la vuelta a ella del obispo san Ramón, expulsado de Barbastro por el obispo de Huesca Esteban en fecha incierta entre 1113 y 1118 , y de Gaufrido, expulsado, a su vez, por el obispo oscense Dodón en 1143. El sucesor de Gaufrido, Guillermo, finalmente, en 1149, tras estar en Roda seis años, trasladó la sede episcopal a la recién conquistada Lérida. Véase al respecto R. de Huesca, Teatro histórico de las Iglesias, pp. 136, 196-197 y 205, y J.C. Boix, De Roda a Lleida, en particular p. 106.

${ }^{19}$ La posibilidad de que el poema fuera compuesto en Roda ya fue sugerida por M. Coll i Alentorn, La historiografia de Catalunya, pp. 46-47, aunque "la reconquesta de Barbastre primer, i sobretot la de Lleida després, havien de desplaçar els centres espirituals i polítics de la contrada vers zones més riques i populoses, i això havia de fer decaure ràpidament la influència i l'activitat d'Alaó i de Roda, convertits des d'aleshores en estoigs de pedra venerable que ens han conservat gelosament els fruits de llur extingida vitalitat". D. Porta, El poema de Roda, p. 307, cree también que "es necesario admitir la posibilidad de su origen rotense", puesto que "Ramón Berenguer IV fue devoto de esa sede; confirmó las numerosas donaciones otorgadas por Ramiro II y, sobre todo, engrandeció la diócesis mediante la incorporación de las tierras leridanas reconquistadas por él; bajo su gobierno, la vieja aspiración de los obispos rotenses de trasladar la sede a Lérida tuvo plena efectividad".

${ }^{20}$ Desde N. Antonio, Bibliotheca Hispana Vetus, t. II, p. 20, núm. 71.

${ }^{21}$ Así lo considera, por ejemplo, J. Villanueva, Viage literario, p. 199.

${ }^{22}$ Méritos, a veces, velados por la insatisfactoria edición de algunos de sus textos publicados. Y también hay que tener en cuenta que la mayoría de los documentos producidos por la antigua sede 
descartar también a Arnaldo como su autor. Con todo, la presencia de escribanos de su categoría permite suponer que una composición de las características de nuestro poema era perfectamente posible en la recóndita catedral de San Vicente de Roda, de donde procede también el Chronicon Rotense, que no olvida evocar la figura del conde barcelonés ${ }^{23}$.

Y digo que debemos descartar a Arnaldo de Coll, porque, a mediados del siglo XII, este clérigo sería todavía muy joven, particularidad que en nada impide, por supuesto, que ya tuviese la suficiente capacidad para componer poemas, aunque la notable calidad del que nos ocupa me lleve a pensar, preferentemente, en un autor más maduro. Arnaldo de Coll era ya canónigo de Roda en $1145^{24}$ y aparece por última vez documentado en $1202^{25}$. Es evidente, pues, que en 1149 era todavía un joven clérigo.

Pero hay una razón de más peso para excluirlo como autor, a él y a otro cualquiera que fuera de Roda o de su entorno. Como la data de la copia, tal como he avanzado, hay que situarla a finales del siglo XII, se realizaría en vida todavía de Arnaldo de Coll. Así pues, el amanuense del poema hubiera tenido la posibilidad de consultar sus dudas al autor y éste le hubiera podido no sólo corregir los errores, sino también completar los espacios en blanco, lo que no sucedió. Por todo ello, aunque la autoría de un canónigo rotense podría ser posible, aun descartando la figura de Arnaldo de Coll, no la veo en absoluto probable.

La cercanía de la fecha de la copia y la de la composición parecen abogar, por el contrario, precisamente por el motivo que acabo de aducir, por un origen distinto de una y otra actividad al no haber contacto entre autor y amanuense, $\mathrm{y}$, al ser éste, indudablemente, rotense. Si, además, como estoy tentado de creer, el poema se compuso acto seguido de la victoria ilerdense, como respuesta inmediata a la conmoción que representaría un éxito militar tan notable, el poeta podría ser un miembro del séquito del conde de Barcelona. Y, ante esta posibilidad, no se me ocurre mejor hipótesis que pensar, de nuevo, en su fiel escribano, el canónigo Poncio, dotado, más aún que Arnaldo de Coll, de una capacidad expresiva y literaria perfectamente probada en la notable cantidad de documentos de naturaleza jurídica salidos de su pluma ${ }^{26}$. Y, en efecto, estoy muy inclinado a suponerlo así. Que después el poema fuera copiado, a medias, en un códice de la catedral ribagorzana, continúa explicándose bien por los beneficios ciertos que la población cristiana de la zona obtuvo de la victoria del conde de Barcelona en Lérida, aplaudido, por lo demás, como hemos visto, en un amplio radio de territorios cristianos. Y, además, esta circunstancia explicaría mejor que la copia del poema en el códice rotense contenga errores y sea incompleto, lo que, como mínimo, nos obliga a pensar, como he dicho, que el copista no estaba cerca del autor.

Sea como sea, el texto, conservado de manera fragmentaria, por interrupción de su copia, ha sido editado en diversas ocasiones, pero nunca con total corrección. Fue dado a conocer, por vez primera, que yo sepa, por el gran estudioso valenciano el P. Jaime Villanueva (Vill. $)^{27}$, de tan clara inteligencia como vivaz pluma, y siempre de gran erudición y excelente criterio, aunque, en esta ocasión, haciendo realidad el

ribagorzana está todavía pendiente de clasificación y sin posibilidad de consulta por parte de los investigadores, por lo que otros escribas quizá igualmente capacitados nos son todavía desconocidos.

${ }^{23}$ Véase la nota 15.

${ }^{24}$ Véase F. Castillón, La fraternidad medieval de Roda, doc. 7, escrito por Arnaldus canonicus de Rota (...) “per iussionem canonici Stephani”. Es posible, pues, que el canónigo Esteban, por cuyo mandato escribe, fuera su maestro.

${ }^{25}$ F. Castillón, La fraternidad medieval de Roda, doc. 25.

26 J. Alturo, El canonge de Barcelona.

27 J. Villanueva, Viage literario, p. 173. 
verso horaciano quandoque bonus dormitat Homerus, cometió algún desliz que se ha mantenido en ediciones más modernas que dependen de la suya.

Su segundo editor fue, por lo que yo conozco, Manuel Milà i Fontanals (Mil.), quien sólo se ocupó del poema tangencialmente y lo editó en una nota a pie de página $^{28}$.

También trató de este poema José Amador de los Ríos (Ama.), que ofreció una versión no del todo exacta ${ }^{29}$, fielmente reproducida, a su vez, por Salvador Martínez $(\text { Mar. })^{30}$, que no corrige un evidente error de imprenta orben e introduce otro nuevo tempuit, y también lo editó y tradujo D. Porta (Por.), que extrajo, como he dicho, casi todo lo que es posible deducir de su contexto, si bien no acertara tampoco, según mi parecer, en una restitución textual completamente fiable. En catalán disponemos también de la bella versión realizada por F. Soldevila ${ }^{31}$ a partir del texto latino establecido por el P. Villanueva.

En resumen, pues, barrunto la posibilidad de que su autor fuera un miembro del entorno más cercano del conde de Barcelona, tal vez su notario y canónigo de Barcelona Poncio, quien lo compondría inmediatamente después de la conquista de Lérida en 1149, y cuya copia pienso que se realizó, en efecto, en Roda en el tránsito del siglo XII al XIII. He aquí, por otra parte, la lectura que propongo del poema a partir de la consulta directa del códice de Roda $(R)^{32}$ :

Fulgent noua per orbem gaudia.

Noua mundum replet leticia,

unde Christo regi sit gloria.

Nouus solis emicat radius

nitens omnis ${ }^{\text {a }}$ sidere clarius, cui non est similis alius.

Cedunt $^{\mathrm{b}}$ ecce falanges hostium.

Nullus pauet hostilem $<$ gladium $>^{c}$.

Tempnit ${ }^{\mathrm{d}}$ quisque sibi contrarium.

Fracta $^{\mathrm{e}}$ cadunt septa ${ }^{\mathrm{f}}$ gentilium, solidantur signa fidelium

per te, comes Barchinonensium.

Idem princeps Aragonensium,

dux Tortose, rex Illerdensium

penestrasti regale solium.

Psallat Deo celi milicia.

\footnotetext{
${ }^{28}$ Véanse sus Observaciones sobre la poesía popular, núm. 3, p. 64. Sobre el maestro de D. Marcelino Menéndez Pelayo, su máximo especialista es M. Jorba; baste citar su libro L'obra crítica $i$ erudita de M. Milà, donde se pone de relieve la gran preparación, incluso en latín, de Milà. Con todo, como simple anécdota, recordemos lo que cuenta de él el gran J.M. de Sagarra, Memòries, p. 226: "És rigorosament autèntic que don Manuel prestà al meu pare un llibre d'estudi que com a senyal de lectura mantenia entre plana i plana una arengada de debò".

29 J. Amador de los Ríos, Historia crítica de la literatura, p. 347.

30 S. Martínez, Épica románica en Cataluña, p. 46.

${ }^{31}$ F. Soldevila, Ramon Berenguer IV, pp. 61-63.

32 En el aparato crítico excluyo las variantes meramente ortográficas y en la edición respeto la ortografía del manuscrito.
} 
Quod nequid humana facundia

soluat Christo celestis curia.

O quam mira Dei $<$ pietas $>^{g}$

${ }^{a}$ omni Vill. Mil. Ama. Por. Mar. ${ }^{\text {b }}$ Cedent Mil. ${ }^{\text {c }}$ gladium om. R Vill., $d u$ bitanter coniec. Mil. Por., certe rest. Ama. Mar. ${ }^{\mathrm{d}}$ Tempuit Mar. ${ }^{\mathrm{e}}$ Tracta Vill. Ama. Mar. ${ }^{\mathrm{f}}$ sepcies $R$ Por., septa corr. Vill. Mil. Ama. Mar. ${ }^{\mathrm{g}}$ pietas deest in $R$.

Ya se ve, pues, que, en mi edición, me mantengo muy fiel a las lecturas que presenta el manuscrito, del que sólo me aparto en septies, que creo error por septa, y en un par más de conjeturas que permiten completar algo el poema trunco. Me ha parecido, incluso, que se debe respetar la lectura omnis que presenta el códice en el verso 5. Todos los editores anteriores corrigen esta palabra en omni, sin duda, por considerar que la $s$ de omnis se debe a una diplografía motivada por la $s$ de sidere que le sigue. Si aceptamos la enmienda, hay que entender el texto así: Un nuevo rayo del sol brilla refulgente con más claridad que toda constelación. En cambio si mantenemos la lección del manuscrito, omnis actuaría como un predicativo de radius y, a mi entender, daría así más fuerza y mayor expresividad a los versos: Un nuevo rayo del sol brilla, refulgente todo él, con más claridad que una constelación. Sin ninguna duda, métrica y textualmente, queda incompleto el verso 8, nullus pauet hostilem, que, tal como conjeturó dubitativamente Milá y otros, de manera independiente y más segura, hay que completar con un gladium, como precisa el metro, exige la rima y sugiere el sentido. El verso 9 comienza, por supuesto, con tempnit y el 10 con fracta $^{33}$, exactamente como está escrito en el códice, sin que haya necesidad, a mi modo de ver, de introducir ninguna corrección. En este mismo verso conviene, sin embargo, enmendar la variante del códice sepcies por septa, por carecer aquella de sentido, a pesar de las consideraciones de Porta, en nada convincentes ${ }^{34}$, entre otros motivos, porque, desde el punto de vista de la métrica, la inclusión de septies daría al verso una sílaba de más puesto que todos son decasílabos. El último verso de nuestro poema tal vez acabara con la palabra pietas, que restituyo como mera posibilidad.

En definitiva, nuestro poema constituye un escaso ejemplo, junto con el canto fúnebre en honor también de Ramón Berenguer IV, Mentem meam ledit dolor, de poesía latina de carácter civil, cuyos antecedentes más inmediatos hay que buscarlos en algunas composiciones del abad Oliba.

\section{BIBLIOGRAFÍA CITADA}

Alturo, Jesús, La historiografia catalana del període primitiu, en Balcells, A. (coord.), Història de la historiografia catalana: Jornades Científiques de l'Institut d'Estudis Catalans, Secció Històrico-Arqueològica: Barcelona 23-25 d'octubre de 2003, Barcelona, Institut d'Estudis Catalans, 2004, pp. 19-38. Alturo, Jesús, El canonge de Barcelona i notari comtal Ponç, en prensa.

Amador de los Ríos, José, Historia crítica de la literatura española, vol. II, Madrid, Imp. José Rodríguez, 1862.

${ }^{33}$ Recordemos, por lo demás, que en el Chronicon Barcinonense se dice de nuestro conde: qui claustra Hispaniae fregit; véase J. Villanueva, Viage literario, y téngase también presente el verso Marte, fame fregi mauros, dum tempore degi escrito en un letrero de su sepulcro, citado en la nota 2.

${ }^{34}$ D. Porta, El poema de Roda, p. 298. 
Antonio, Nicolás, Bibliotheca Hispana Vetus, vol. II, Matriti, Apud viduam et heredes Ioachimi Ibarrae, 1788.

Appelt, Heirich; Herkenrath, Rainer Maria; Koch, Walter (eds.), Friderici I. Diplomata, Hannover, Hahnsche Buchhandlung, 1979. (Monumenta Germaniae Historica. Die Urkunden der deutschen Könige und Kaiser; 10-2).

Arco, Ricardo del, Referencias a acontecimientos históricos en las datas de documentos aragoneses de los siglos XI y XII, "Estudios de Edad Media de la Corona de Aragón" 3 (1947-1948), pp. 291-354.

Barrau Dihigo, Louis; Massó Torrents, Jaume (eds.), Gesta comitum Barcinonensium, Barcelona, Fundació Concepció Rabell i Civils - Vidua Romaguera, 1925.

Bautista, Francisco, Breve historiografía: Listas regias y Anales en la Península Ibérica (siglos VII-XII), "Talia Dixit" 4 (2009), pp. 113-190.

Bofarull, Próspero de, Los condes de Barcelona vindicados, y cronología y genealogía de los reyes de España considerados como soberanos independientes de su Marca, vol. II, Barcelona, J. Oliveres, 1836.

Boix, Jordi C., De Roda a Lleida: la fi d'un somni heroic, [Vielha], Institut d'Estudis del Baix Cinca, 1998.

Castillón, Francisco, La fraternidad medieval de Roda de Isábena (Huesca), “Argensola" 105 (1991), pp. 11-120.

Cingolani, Stefano Maria, Gestes dels Comtes de Barcelona i Reis d'Aragó, València, Universitat de València, 2008.

Coll i Alentorn, Miquel, La historiografia de Catalunya en el període primitiu, "Estudis Romànics" 3 (1951-1952), pp. 139-196. [Reimp. en Coll i Alentorn, Miquel, Historiografía, Barcelona, 1991. Textos i estudis de cultura catalana, 21; Obres de Miquel Coll i Alentorn, 1, pp. 11-62].

Huesca, Ramón de, Teatro histórico de las Iglesias del Reyno de Aragón, vol. IX, De las Iglesias Catedrales y Diócesis de Roda y Barbastro, Zaragoza, Oficina de Miedes, 1807.

Jorba, Manuel, L'obra crítica i erudita de M. Milà i Fontanals, Barcelona, Curial Abadia de Montserrat, 1989.

Martín Duque, Angel, Colección diplomática del monasterio de San Victorián de Sobrarbe (1000-1219), Zaragoza, Universidad de Zaragoza, 2004.

Martínez, Salvador, Épica románica en Cataluña. Reliquias de una tradición latina, en Studia in honorem prof. M. de Riquer, vol. IV, Barcelona, Quaderns crema, 1988, pp. 25-68.

Merino, Antolín; Canal, José de la, España Sagrada, vol. XLIII, Madrid, Imp. de Antonio Marin, 1819.

Miquel Rosell, Francisco, Liber Feudorum Maior. Cartulario real que se conserva en el Archivo de la Corona de Aragón, vol. II, Barcelona, Consejo Superior de Investigaciones Científicas, 1947.

Milá y Fontanals, Manuel, Observaciones sobre la poesía popular con muestras de romances catalanes inéditos, Barcelona, Imp. de Narciso Ramírez, 1853.

Nicolau d'Olwer, Lluís, L'escola poètica de Ripoll en els segles X-XIII, "Anuari de l'Institut d'Estudis Catalans" 6 (1915-1920), pp. 3-84.

Porta, Dolores, El poema de Roda en honor de Ramón Berenguer IV, "Argensola" 44 (1960), pp. 297-310.

Pujades, Gerónimo, Crónica Universal del Principado de Cataluña, escrita a principios del siglo XVII, Tercera parte, vol. VIII, Barcelona, Imprenta de José Torner, 1832.

Rodríguez Bernal, Francisco, Ramón Berenguer IV y su tiempo: una aproximación social, "Cuadernos del CEHIMO" 35 (2009), pp. 13-26. 
Roure, Alfons, Vassall d'amor. Vida turmentada de Ramon Berenguer IV comte de Barcelona, Barcelona, Imp. Garrofé, 1924.

Ruiz-Domènec, José Enrique, España, una nueva historia, Madrid, Gredos, 2009.

Savall, Jordi, Mentem meam ledit dolor. Anonymous, en The Forgotten Kingdon, [S.1.] Alia Vox, 2009, (1 Cd-Rom).

Sagarra, Josep Maria de, Memòries, vol. I, València, Tres i Quatre, 2003.

Schramm, Percy E., Ramon Berenguer IV, en Schramm, Percy E., Els primers comtesreis, Barcelona, Vicens-Vives, 1960, pp. 9-51.

Soldevila, Ferran, Ramon Berenguer IV el Sant, Barcelona, Barcino, 1955.

Villanueva, Jaime, Viage literario a las iglesias de España, vol. XV, Madrid, Impr. de la Real Academia de la Historia a cargo de Jose Rodriguez, 1851.

Fecha de recepción del artículo: enero 2011

Fecha de aceptación y versión final: mayo 2011 